\title{
Unilateral Pedicular Fixation and Transforaminal lumbar Interbody Fusion in the treatment of Degenerative Lumbar Disc Disease
}

\author{
Adel A. Abdel Aziz, Ahmed M. Ahmed Othman, Mohamed M. Azmy \\ and Mostafa A. Mostafa \\ Department of Orthopedic, El-Minia Faculty of Medicine
}

\begin{abstract}
Introduction: For a couple of decades particularly, both the increase in average age throughout the world and the developments in diagnostic technologies have gradually increased the importance of lumbar degenerative diseases in the practice of neurosurgery. Aim of the Work: The aim of this work is to evaluate the role of transforaminal lumbar interbody fusion with a single oblique cage and unilateral pedicular fixation in the treatment of degenerative lumbar disc disease. The results were recorded, tabulated \& finally analyzed. Patients \& Methods: This prospective study included thirty patients with degenerative lumbar disc diseases treated by lumbar interbody fusion using unilateral pedicular fixation. All surgeries were performed in the spine unit at Minia University Hospital, El Minia, Egypt. Patients were followed up for at least 6 months, and all of them were consented. Results: Follow up: All patients will be followed up closely; day after day for wound care until the removal of stiches after 15 days, clinical examination and radiographies will be performed at one month, three months, 6 months, 9 months and one year intervals after the operation. Conclusion: 1- Unilateral PS instrumented TLIF is a viable treatment option for treating Degenerative disc disease as it successfully achieves: a- Direct and indirect decompression and hence addressing the main pathology which is the nerve root compression. b-One stage circumferential fusion. 2-UNI-TLIF produces good results especially in terms of operative time, blood loss, implant costs, and hospital time for single or double-level diseases. 3- UNITLIF should be extended to include other inclusion criteria. 4-An improved study design and longer period of follow-up are still needed to confirm this effect.

Keywords: Unilateral Pedicular Fixation and Transforaminal, lumbar Interbody Fusion
\end{abstract}

\section{Introduction}

For a couple of decades particularly, both the increase in average age throughout the world and the developments in diagnostic technologies have gradually increased the importance of lumbar degenerative diseases in the practice of neurosurgery. The most widely accepted algorithm in the literature is medical treatment for an appropriate period of time, physical therapy and minimally invasive pain-relieving therapies, if necessary, followed by surgical interventions. The most common surgical intervention is the decompression of neural elements followed by pedicle screw fixation (PSF) for fusion. Together with the definition of transforaminal lumbar interbody fusion (TLIF) by Harms, particularly after 1990s, TLIF procedures are added to the pedicle screw practices, and many authors in the literature reported that a more strong and reliable fusion could be possible with this technique ${ }^{(1,2)}$.

In recent years, there is an ongoing discussion in the literature on whether the pedicle screw fixation implemented together with TLIF in order to have a more strong, reliable, less invasive, less expensive fusion with less complications, should be implemented unilaterally or bilaterally ${ }^{(3,4)}$. In fact, traditionally practiced bilateral PSF and instrumentation performed together with TLIF can be regarded as a convenient procedure for a reliable, effective and biomechanically sufficient fusion ${ }^{(5-8)}$. 
On the other hand, it is reported that extreme rigidity caused by bilateral screw fixation lead to development of adjacent segment disease (ASD). Subsidence of bone graft and decline in bone mineral cause a decrease in fusion rates. ${ }^{(5-7,}$ 9-12). Likewise, it is reported in the literature that unilateral pedicle fixation with the TLIF procedure has many advantages compared to bilateral PSF.

The main advantages are shorter operation time, less invasive surgical procedure, less blood loss, less postoperative pain, shorter hospital stay, less cost and less comorbidities of $\mathrm{ASD}^{(7,13-16)}$.

Nonetheless, some researchers state that unilateral screw fixation decreases stability and rigidity related to the axial rotational resistance and lateral bending, which is seen together with less fusion, leading to more cage migration cases and that it is not suitable for the stabilization of long segments [2, 5, 9, 12, 17-20]. Therefore, it is obvious that a consensus is yet to be reached in the literature as regards which instrumentation should be used on which patient and how. In their study, Chen D-j et al., ${ }^{[21]}(2018)$ examined the clinical and radiological follow up and results of their patients undergoing fusion procedure by unilateral PSF along with TLIF. They aimed at contributing to the discussions in the literature by analyzing the results together with previously published studies ${ }^{[21]}$ (2018).

\section{Aim of the Work}

The aim of this work is to evaluate the role of transforaminal lumbar interbody fusion with a single oblique cage and unilateral pedicular fixation in the treatment of degenerative lumbar disc disease. The results were recorded, tabulated \& finally analyzed

\section{Patients \& Methods Introduction}

This prospective study included thirty patients with degenerative lumbar disc diseases treated by lumbar interbody fusion using unilateral pedicular fixation. All surgeries were performed in the spine unit at Minia University Hospital, El Minia, Egypt. Patients were followed up for at least 6 months, and all of them were consented.

\section{Ethical consideration:}

The study was approved by the Ethical Committee of the Faculty of medicine Minia University. Informed consent with risk explanation was obtained from all participating patients. Every patient had the right to refuse participation in the study without affecting the service or the clinical management. All of the patients were free to ask any question about the study.

\section{Confidentiality:}

The confidentiality of all participants admitted to this study was protected to the fullest extent possible. The study participants were not identified by name in any report or publication resulting from data collected in this study.

\section{Research statement:}

Ethical aspects whether substantial or procedural were implicated in this study. Before participants were admitted in this study, the purpose and nature of the study as well as the risks were explained to them. The participants agreed that they understood the investigational nature of the study, its inherent risks and benefits, their rights to terminate their participation in this study without affecting their rights in having proper health care in the study site, whom to contact with questions regarding the study and that they were freely given an informed consent to to participate in this study.

\section{Results}

\section{Follow up:}

All patients will be followed up closely; day after day for wound care until the removal of stiches after 15 days, clinical examination and radiographies will be performed at one month, three months, 6 months, 9 months and one year intervals after the operation. 


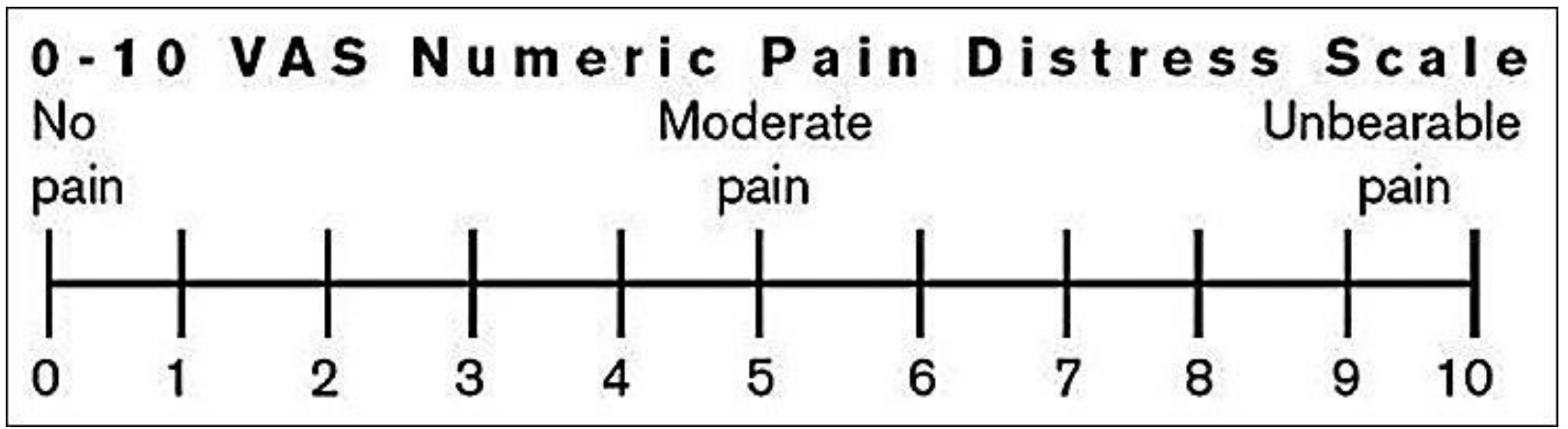

Pain assessment : by Visual Analogue Score ( VAS )(76) for back pain (if present ) at preoperative time, postoperative time, during the follow up and at the end of the study. The pain visual analog scale is self-completed by the respondent. The respondent is asked to place a line perpendicular to the VAS line at the point that represe;nts their pain intensity. After the patient has marked, using a ruler, the score is determined by measuring the distance $(\mathrm{mm})$ on the $10-\mathrm{cm}$ line between the "no pain" anchor and the patient's mark. The scores can be from 0-100. A higher score indicates greater pain intensity.
Based on the distribution of pain VAS scores in postsurgical patients, the following cut points on the pain VAS have been recommended.

- No pain (0 -4 mm)

- Mild pain (5-44 mm)

- Moderate pain (45-74 mm)

- $\quad$ Severe pain (75-100 mm)

The VAS scores of our patients showed dramatic improvement over time starting with a preoperative mean VAS back of 9 and a mean VAS back score at 6 months follow up of 1.366 Figure ().

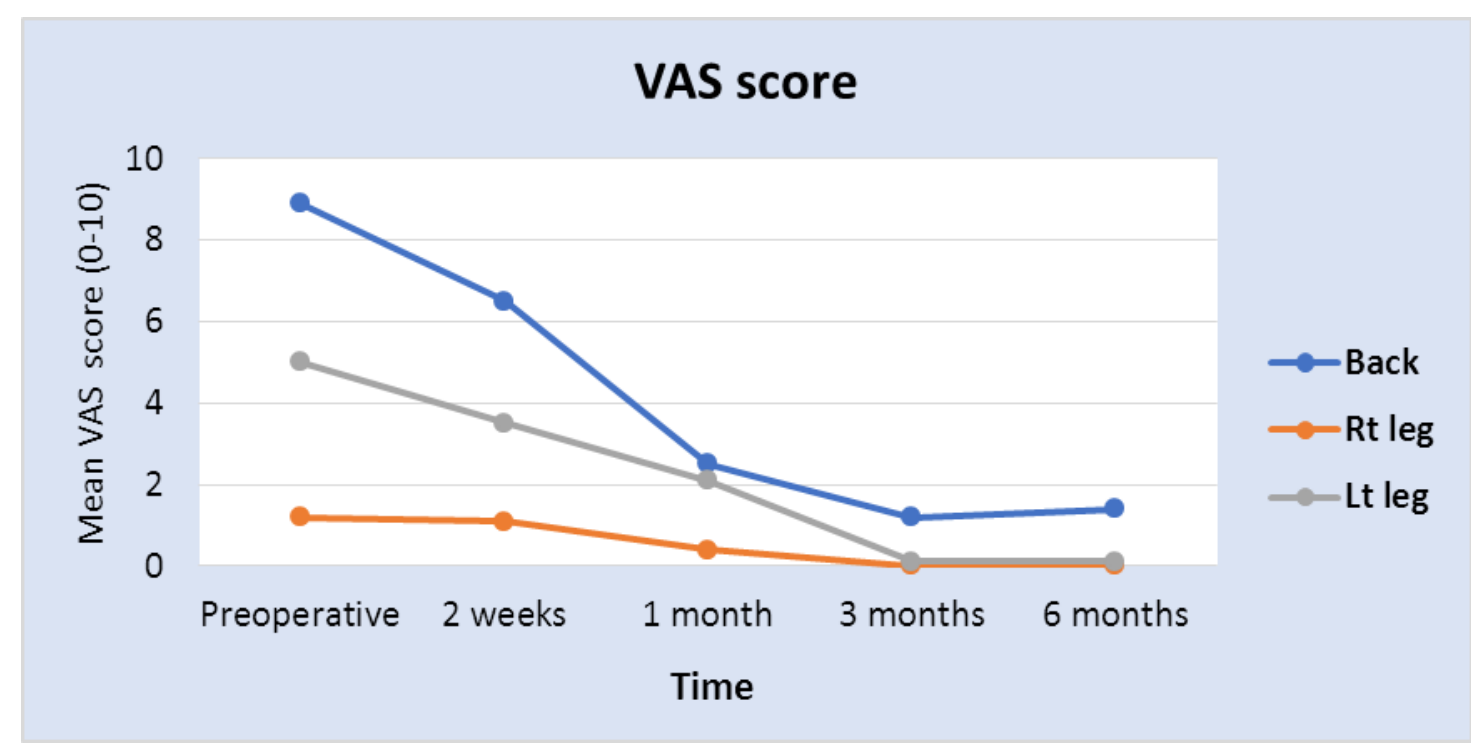

Figure (): A line chart showing the change in the VAS scores over time

\section{Radiological assessment:}

Plain X-ray.

MRI when required.

CT to assess the success of interbody fusion according to the Brantigan, Steffee, Fraser(BSF) classification (77) 


\section{Discussion}

The advantage of anterior column support and fusion in addition to pedicle fixation in patients with degenerative spinal disorders has become increasingly clear. With the increase in popularity of this treatment, a variety of techniques have been used to achieve the goal of anterior column support, fusion, and segmental instrumentation (Omar El Farouk Ahmed et al., 2020).

Posterior lumbar interbody fusion has been used since the late 1940s in the treatment of degenerative lumbar spine. The risk of PLIF approach-related injuries is well recognized $^{(2)}$.

Anterior lumbar interbody fusion represents another alternative to a 360-degree spinal fusion but requires a separate anterior approach. In 1982, with the rationale of offering a secure fusion in a one stage operation, Harms and Rolinger ${ }^{(2)}$ pioneered a modified PLIF technique called transforaminal lumbar interbody fusion (TLIF) that can achieve a one stage circumferential fusion and achieve direct decompression via hemi-laminectomy and inferior facetectomy ,and indirect decompression through restoration of the disc height (that decreases as a result of degenerative disc disease) and correction of deformity (that results from spondylolistheses) .

Transforaminal lumbar interbody fusion (TLIF) is traditionally performed with bilateral pedicle screw (PS) fixation, and there are only a small number of case reports of unilateral instrumented TLIF and its efficiency. However, the stress shielding caused by rigid internal fixation is thought to lead to osteopenia and degeneration of adjacent segments, theatre times, intraoperative complications and costs are increased when pedicle screw fixation is added in bilateral technique (3) (Mario Cahueque et al., 2019).

\section{On analysis of our results}

Our patients were assessed radiologically via normal X-rays and MRI (preoperatively only), but postoperatively were followed up with X-rays only and MRI or CT only when needed, and clinically via Visual analogue scale (VAS) and modified Prolo score (mProlo) preoperatively and postoperatively.

The mProlo score was chosen instead of the Oswestery Disability Index (ODI ) as it is way simpler than the ODI and was more applicable on our population because it has less number of questions that cover nearly the same aspects of the ODI score without the added complexity and also the mProlo score has been used successfully in differrent studies to assess the success of interbody fusion as In 2000, when Brantigan et al., ${ }^{(4)}$ modified the original scale(developed in 1986) to our current version in amulticenter-2-year retrospective randomized trial in which they administered a protocol that was created in the 1990s and approved by the Food and Drug Administration (FDA) in 1999 in order to introduce a surgical device (I/F carbon cage) for posterior lumbar interbody fusion. The authors declined using common tools to assess the LBP (e.g., the ODI, RMDQ, etc.), yet they administered the PS because it was more useful to compare data from surgical studies carried out at different times.

\section{Conclusion}

In conclusion:

1- Unilateral PS instrumented TLIF is a viable treatment option for treating Degenerative disc disease as it successfully achieves:

a- Direct and indirect decompression and hence addressing the main pathology which is the nerve root compression.

b- One stage circumferential fusion.

2- UNI-TLIF produces good results especially in terms of operative time, blood loss, implant costs, and hospital time for single or double-level diseases.

3- UNI-TLIF should be extended to include other inclusion criteria.

4- An improved study design and longer period of follow-up are still needed to confirm this effect.

\section{References}

1. Harms J, Rolinger HJZfOuiG. A onestager procedure in operative treatment 
of spondylolistheses: dorsal tractionreposition and anterior fusion (author's transl). 1982;120(3):343-7.

2. Hu X-Q, Wu X-L, Xu C, Zheng X-H, Jin Y-L, Wu L-J, et al., A systematic review and meta-analysis of unilateral versus bilateral pedicle screw fixation in trans-foraminal lumbar interbody fusion. 2014; 9(1)

3. Aoki $Y$, Yamagata $M$, Nakajima $F$, Ikeda Y, Shimizu K, Yoshihara M, et al., Examining risk factors for posterior migration of fusion cages following transforaminal lumbar interbody fusion: a possible limitation of unilateral pedicle screw fixation. 2010;13(3):381-7.

4. Li Z, Liu F, Liu S, Chen Z, Jiang C, Feng Z, et al., Miniopen transforaminal lumbar interbody fusion with unilateral fixation: A comparison between ipsilateral and contralateral reherniation. 2016;2016.

5. Shen X, Zhang H, Gu X, Gu G, Zhou $X, \mathrm{He}$ SJJocn. Unilateral versus bilateral pedicle screw instrumentation for single-level minimally invasive transforaminal lumbar interbody fusion. 2014;21(9):1612-6.

6. Yuan C, Chen K, Zhang H, Zhang H, $\mathrm{He}$ SJCn, neurosurgery. Unilateral versus bila-teral pedicle screw fixation in lumbar interbody fusion: a metaanalysis of complication and fusion rate. 2014;117:28-32.

7. Li $\mathrm{X}, \mathrm{Lv} \mathrm{C}$, Yan TJMsmimjoe, research c. Unilateral versus bilateral pedicle screw fixation for degenerative lumbar diseases: A meta-analysis of 10 randomized controlled trials. 2015; $21: 782$.

8. Isik HS, Okutan O, Yildirim $\mathrm{T}$, Akpinar E, Yilmaz AJTN. Comparison of Unilateral versus Bilateral Pedicle Screw Fixation in Transforaminal Lumbar Interbody Fusion for Single Level Lumbar Degenerative Diseases and Review of Literature. 2017:1.

9. Yücesoy $\mathrm{K}$, Yüksel $\mathrm{KZ}$, Baek $\mathrm{S}$, Sonntag VK, Crawford NRJJoNS. Biomechanics of unilateral compared with bilateral lumbar pedicle screw fixation for stabilization of unilateral vertebral disease. 2008;8(1):44-51.
10. Xue H, Tu Y, Cai MJTSJ. Comparison of unilateral versus bilateral instrumented transforaminal lumbar interbody fusion in degenerative lumbar diseases. 2012;12(3):209-15.

11. Phan K, Leung V, Scherman DB, Tan AR, Rao PJ, Mobbs RJJJoCN. Bilateral versus unilateral instrumenttation in spinal surgery: systematic review and trial sequential analysis of prospective studies. 2016;30:15-23.

12. Yang S-D, Chen Q, Ding W-Y, Zhao J-Q, Zhang Y-Z, Shen Y, et al., Unilateral pedicle screw fixation with bone graft vs. bilateral pedicle screw fixation with bone graft or cage: A comparative study. 2016;22:890.

13. Rihn JAJTSJ. Commentary: is bilateral pedicle screw fixation necessary when performing a transforaminal lumbar interbody fusion? An analysis of clinical outcomes, radiographic outcomes, and cost. 2012;12(3):216-7.

14. Kim T-H, Lee BH, Moon S-H, Lee S$\mathrm{H}$, Lee H-MJTSJ. Comparison of adjacent segment degeneration after successful posterolateral fusion with unilateral or bilateral pedicle screw instrumentation: a minimum 10-year follow-up. 2013;13(10):1208-16.

15. Chen C, Cao X, Zou L, Hao G, Zhou Z, Zhang GJJoos, et al., Minimally invasive unilateral versus bilateral technique in performing singlesegment pedicle screw fixation and lumbar interbody fusion. 2015;10 (1): 112 .

16. Zhao C-Q, Ding W, Zhang K, Zhao JJIjoo. Transforaminal lumbar interbody fusion using one diagonal fusion cage with unilateral pedicle screw fixation for treatment of massive lumbar disc herniation. 2016; 50 (5):473.

17. Suk KS, Lee HM, Kim NH, Ha JWJS. Unilateral versus bilateral pedicle screw fixation in lumbar spinal fusion. 2000;25(14):1843-7.

18. Harris BM, Hilibrand AS, Savas PE, Pellegrino A, Vaccaro AR, Siegler S, et al., Transforaminal lumbar interbody fusion: the effect of various instrumentation techniques on the flexibility of the lumbar spine. 2004;29(4):E65-E70. 